\title{
A Secure and High Robust Audio Watermarking System for Copyright Protection
}

\author{
Himeur Yassine \\ Department of electronics \\ University of Jijel \\ Algeria
}

\author{
Boudraa Bachir \\ LCPTS \\ USTHB \\ Algeria
}

\author{
Khelalef Aziz \\ Department of electronics \\ University of Batna \\ Algeria
}

\begin{abstract}
Transform-domain digital audio watermarking has a performance advantage over time-domain watermarking by virtue of the fact that frequency transforms offer better exploitation of the human auditory system (HAS). In this paper, an innovative watermarking scheme for audio signal based on double insertion of the watermark in DWT-DST domain of the host signal is proposed. We are using a gray scale logo image as watermark instead of randomly generated Gaussian noise type watermark. Subjective and objective tests reveal that the proposed watermarking scheme maintains high audio quality and is simultaneously highly robust to different attacks, including MP3 compression, low-pass filtering, amplitude scaling, additive Gaussian noise, reacquisition, cropping, sampling, high pass filtering. Comparison of the proposed algorithm with similar techniques such as Cox et al and Dhar et al, shows the superiority of the proposed scheme in term of robustness and imperceptibility.
\end{abstract}

\section{Keywords}

audio watermarking, DWT, PN-sequence, DST,FRIT, copyright protection, robust watermarking.

\section{INTRODUCTION}

Audio signals in digital form are easily reproducible without any distortion; effective protection techniques have become indispensable. This need has been reinforced by the new means of distribution, such as Internet and by compression methods, such as MPEG-2, AAC and MP3.

The traditional information security technology based on cryptography theory mostly has its limitations. In order to resolve the shortcomings of traditional information security technology, more and more researchers has been starting to study the digital watermarking technology because it can effectively compensate for the deficiencies of the security and protection application of traditional information security technology. Watermarking is a process that embeds data called watermark or digital signature or tag or label into a multimedia object such that the watermark can be detected or extracted later to make an assertion about the object [1]. Digital watermarking technology has many applications in protection, certification, distribution, anti-counterfeit of the digital media and label of the user information. It has become a very important study area in information hiding [2].

Several watermarking techniques have been developed and commercialized. The watermarks produced by those techniques can withstand a number of single attacks such as MPEG, resampling, filtering and quantization. However, the watermarks are easily destroyed if subjected to chopping and multiple attacks Existing audio watermarking systems exploit the irrelevant properties of the human auditory system (HAS) [3]. In particular, HAS is insensitive to small amplitude changes in the time and frequency domains, allowing the addition of weak noise signals (watermarks) to the host audio signal such that the changes are inaudible [4-9]. Frequencydomain techniques, in particular, have been more effective than time-domain techniques since watermarks are added to selected regions in the transformed domain of the host audio signal, such that inaudibility an robustness are maintained [10-12]. This paper, will report on hybrid-transform audio watermarking algorithm. Two powerful transforms are applied on the original audio signal; discrete wavelets transform (DWT), and Discrete Sine Transform (DST) using a double insertion of the watermark.

The paper is organized as below. Section 1 provides an introduction to audio watermarking systems, Section 2 describes transformation techniques, Section 3 discusses the proposed watermark embedding and extraction process and Section 4 presents simulation results and discussion. A brief conclusion with future direction is presented in Section 5 .

\section{TRANSFORMATION TECHNIQUES}

\subsection{Discrete wavelet Transform}

The discrete wavelet transform has received a tremendous amount of interest in many important signal processing applications, including audio and image watermarking [13- 15]. Wavelet transform can be used to decompose a signal into two parts: high frequencies and low frequencies. The low frequencies part is decomposed again into two parts of high and low frequencies. The number of decompositions in this process is usually determined by an application and length of an original signal. The data obtained from the above decomposition are called the DWT coefficients. Moreover, the original signal can be reconstructed from these coefficients. This reconstruction is called the inverse DWT. For more information on Wavelet transform, see [16].

\subsection{Discrete Cosine Transform}

Like other transforms, the Discrete Cosine Transform (DCT) attempts to decorrelate the image data. After decorrelation 
each transform coefficient can be encoded independently without losing compression efficiency. The most common DCT definition of a 1-D sequence of length $\mathrm{N}$ is [17]:

$$
y(k)=w(k) \sum_{n=0}^{N-1} x(n) \cos \frac{\pi(2 n-1)(k-1)}{2 N},
$$

for $k=0,1,2, \ldots, N-1$. Similarly, the inverse transformation is defined as:

$$
x(n)=\sum_{n=0}^{N-1} w(k) y(k) \cos \frac{\pi(2 n-1)(k-1)}{2 N},
$$

for $n=0,1,2, \ldots, N-1$. In both equations (1) and (2) $w(k)$ is defined as:

$$
w(k)=\left\{\begin{array}{lr}
\frac{1}{\sqrt{N}}, & k=0 \\
\sqrt{\frac{2}{N}}, & 1 \leq k \leq N-1
\end{array}\right.
$$

Thus, the first transform coefficient is the average value of the sample sequence. In literature, this value is referred to as the DC Coefficient. All other transform coefficients are called the AC Coefficients.

\subsection{Discrete Sine Transform}

DST is identical to Shifted Discrete Fourier Transform spectra of signals that are certain permutation modifications of the original signal: The DST is defined by the following equation [18]:

$$
y(k)=\sum_{n=0}^{N-1} x(n) \sin \left(\frac{\pi k n}{N+1}\right), k=0, \ldots N-1
$$

It also has a property of energy compaction that can be used for audio watermarking. The Equation (5) calculates the value of the inverse discrete sine transform:

$$
x(n)=\frac{2}{N+1} \sum_{n=0}^{N-1} y(k) \cdot \sin \left(\frac{\pi k n}{N+1}\right),
$$

Where $n=0, \ldots, N-1$

\subsection{Difference between the DCT and the DST}

By applying the DCT to 8 identical values $(x(i)=100$, $i=1,2, \ldots, 8)$ it can see that the DCT compacts all the energy of the data into the single DC coefficient whose value is identical to the values of the data items. Applying the DST to the same eight values, on the other hand, results in seven $\mathrm{AC}$ coefficients whose sum is a wave function that passes through the eight data points but oscillates between the points [19].

So it can say that the DST has compacted the energy input values by using more AC coefficients than the DCT.

This example, with the fact that the DCT produces highly decorrelated coefficients, argues strongly in favor of using the DCT in data compression in contrast to DST.

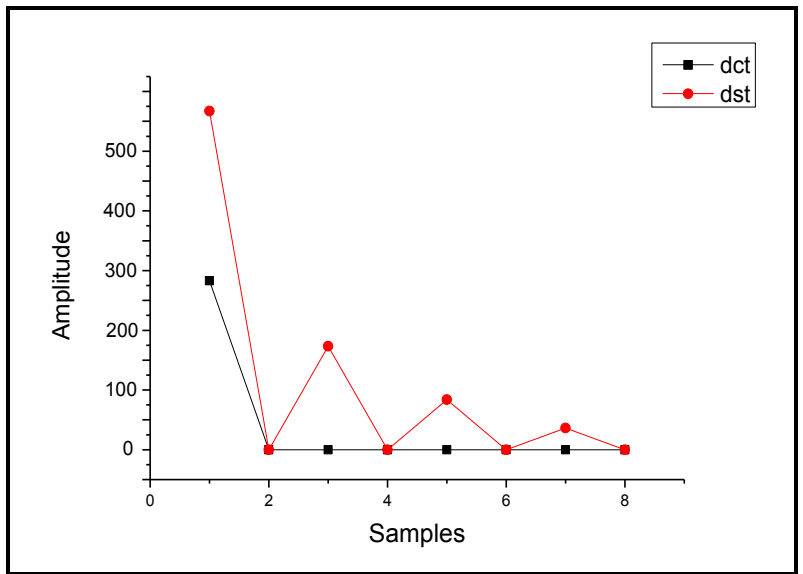

Fig 1: DCT and DST of eight identical values

In watermarking, the insertion of the watermark in the DC coefficients gives a better robustness, but degrades the quality of the watermarked signal. In addition, the DCT does not have many choices for insertion of the watermark, while the DST, as a result of compacting the signal energy over several coefficients gives more choices and therefore more security to watermarking system. In addition, it ensures a good compromise between robustness and imperceptibility.

\subsection{The Finite Ridgelet Transform}

Finite Ridgelet Transform, also known as FRIT, is a kind of invertible, non-redundant transform [20]. To overcome the periodization effect of a finite transform, by using a novel ordering of the FRAT coefficients. Taking the onedimensional wavelet transform on the projections of the FRAT in a special way results in the finite ridgelet transform (FRIT), which is invertible, non-redundant, and computed via fast algorithms?

In 2-D, points and lines are related via the Radon trans- form, thus the wavelet and ridgelet transforms are linked via the Radon transform. More precisely, denote the Radon transform as :

$$
R_{f}(\theta, t)=\int_{R^{2}} f(x) \delta\left(x_{1} \cos \theta+x_{2} \sin \theta-t\right) d x
$$

Then the ridgelet transform is the application of a 1-D wavelet transform to the slices (also referred to as projections) of the Radon transform,

$$
C R T_{f}(a, b, \theta)=\int_{R} \psi_{a, b}(t) R_{f}(\theta, t) d t
$$

So, the advantage of the Finit Ridgelet Transform, is invertible and to compute its inverse transform you must have two information on the original image. Namely; the normalized mean and the structure of wavelet decomposition that are needed for reconstruction. These two information are generated during the transformation process and without this information the reconstruction of the original image cannot be done. In this paper, we propose to use the FRIT as an encrypting system to secure the watermarking algorithm. So, the transformed coefficients are inserted in the host audio signal. 


\section{WATERMARKING EMBEDDING AND EXTRACTION PROCESS}

\subsection{Watermarking embedding process}

The proposed watermark embedding process is shown in Figure 2. The embedding process is implemented in the following seven steps:

1) The original audio signal is first decomposed with the wavelet transform.

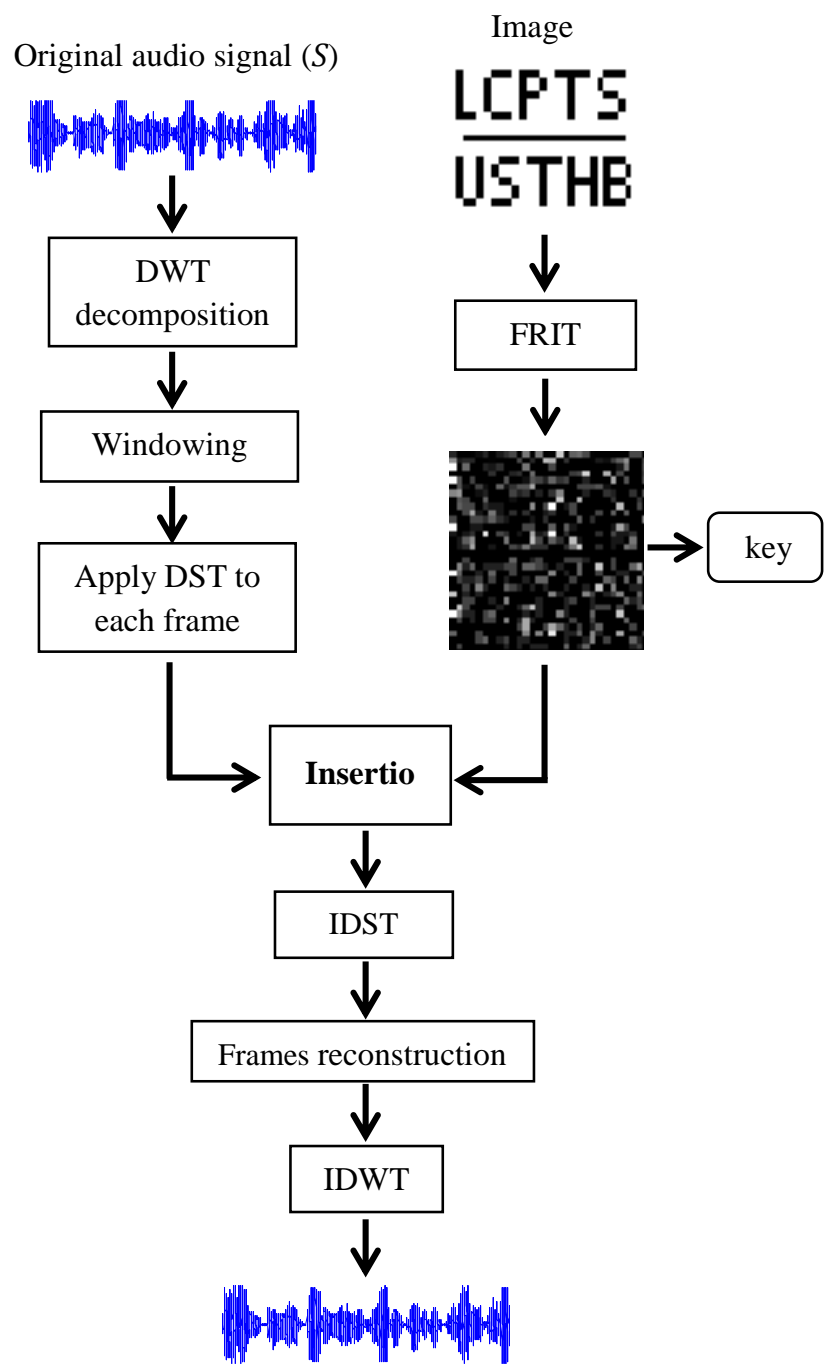

Watermarked signal $\left(S_{w}\right)$

Fig 2: Watermark embedding process

2) The approximation $\left(A_{1}\right)$ and detail $\left(D_{1}\right)$ coefficients are then segmented into non-overlapping frames and transformed with the DST. The watermark is first transformed with the FRIT and the result coefficients are double inserted into the approximation (low frequencies) and detail coefficients (high frequencies). The insertion of the watermark in low frequency presents good robustness against some attacks like mp3 compression, low pass filtering, resampling, requantization, etc, but it doesn't show any robustness against amplification and high pass filtering. So, the double insertion of the watermark in low and high frequency coefficient can give more robustness against these two attacks.
3) Each sample of the watermark is then inserted into a coefficient of each frame using equation (8) [11]. Generally samples of the watermark are inserted in the first five coefficients of each frame. This ensures that the mark is added to the components of the most important perception.

$$
S_{w}=S+\alpha \times W
$$

The value of $\alpha$ denotes the strength of the watermarking and it is called embedding strength, where increasing $\alpha$ increases the robustness of the watermark at the expense of the audio quality. Therefore, it can be chosen such that audio quality is not degraded.

5) Take the inverse DST of the modified DST coefficients to calculate the watermarked high and low frequencies $A^{w}{ }_{1}$ and $D^{w}{ }_{1}$ respectively.

6) At the end, the watermarked audio signal $S_{w}$ is reconstructed using the inverse discrete wavelet transform.

\subsection{Watermarking extraction process}

The proposed watermark extraction process is shown in Fig. 3. It is implemented in the following three steps:

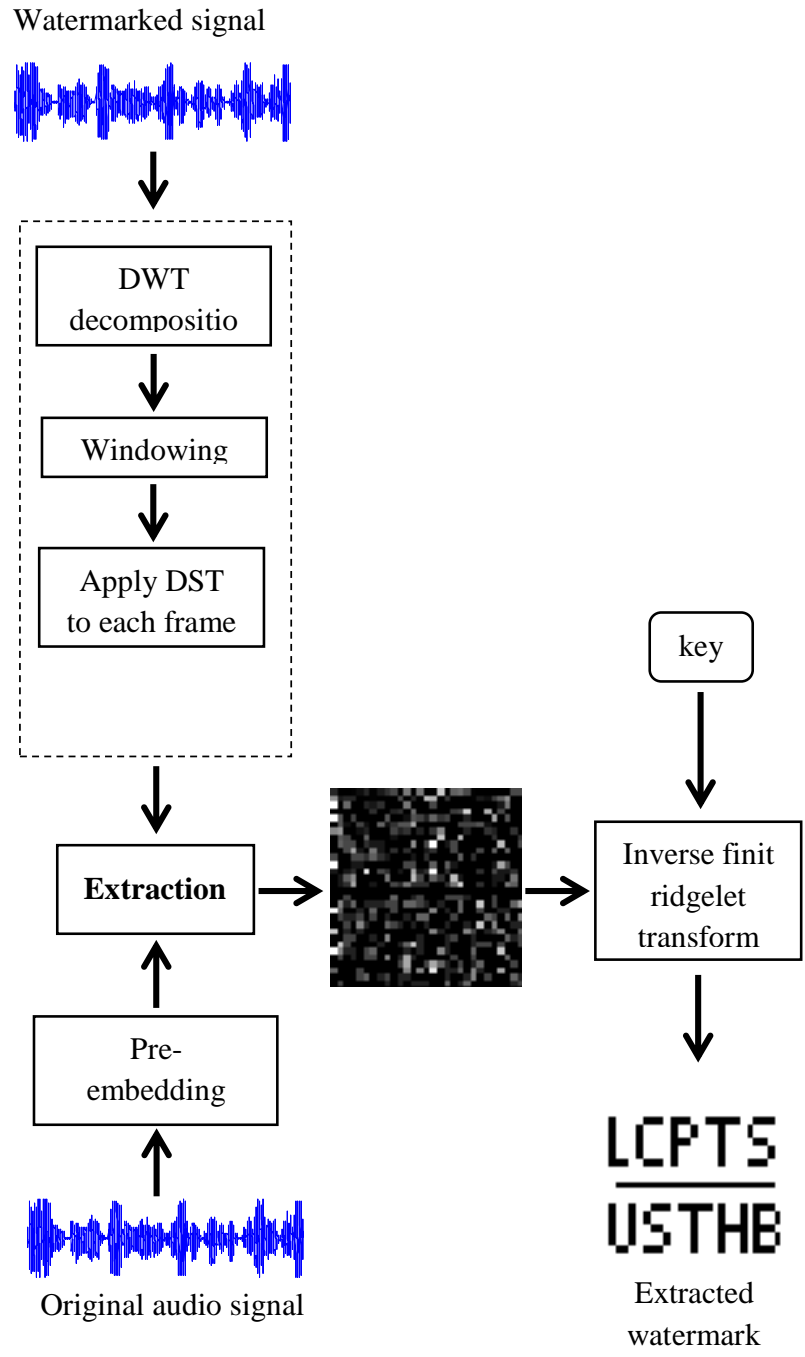

Fig 3: Watermark extraction process 
1) Calculate the DWT of the attacked watermark audio signal.

2) The approximation and detail coefficients $A^{w}{ }_{1}$ and $D^{w}{ }_{1}$ are then segmented into non-overlapping frames and transformed with the DST.

3) The N coefficients of the watermark inserted are extracted from the first frames coefficients of $S w$ have been selected during the insertion phase using the following relation.

$$
W^{\prime}=\frac{S_{w}-S}{\alpha}
$$

The obtained coefficients are than transformed with the inverse FRIT.

Thus, the extraction and transformation operations retrieve the two watermarks included in the coefficients of low and high frequencies. Only the watermark that has suffered the least damage is kept. It will be used as proof of ownership of

\section{SIMULATION RESULTS AND DISCUSSION}

In this section, the performance of the proposed watermarking system is evaluated using eight different types of 16 bit mono audio signals sampled at $44.1 \mathrm{kHz}$ for $\alpha=7$ by considering the frame size of 512 samples. A comparison study has been done between the proposed algorithm, cox's algorithm [11] and Dhar's algorithm [12]. In this paper, we propose to use a binary image as a watermark instead of the $\mathrm{PN}$-sequence used in [11] and [12], to allow a subjective validation of the extracted watermark.

Table 1. Audio signals used in the simulation

\begin{tabular}{|l|l|l|}
\hline Signal & Title & Description \\
\hline S1 & listening & A female voice \\
\hline S2 & Male Voice & A male voice \\
\hline S3 & $\begin{array}{l}\text { Fifth } \\
\text { symphony }\end{array}$ & $\begin{array}{l}\text { the beginning of the Symphony } \\
\text { No. 5 in c minor, Op. 67 written } \\
\text { by Ludwig van Beethoven }\end{array}$ \\
\hline S4 & arabic music & $\begin{array}{l}\text { Arab music, played by traditional } \\
\text { guitar (mandole) }\end{array}$ \\
\hline S5 & Let it be & written by Beatles \\
\hline S6 & Piano & played by Nassib Nassar \\
\hline S7 & Hey Jude & $\begin{array}{l}\text { Played by a Korean traditional } \\
\text { musical instrument called the } \\
\text { gayageum }\end{array}$ \\
\hline S8 & $\begin{array}{l}\text { Classical } \\
\text { music }\end{array}$ & violin music \\
\hline
\end{tabular}

\section{IV.1 Imperceptibility Test}

Imperceptibility tests are essential to perceptual quality assessment, since the ultimate judgment is made by human acoustic perception. Informal listening reveals excellent imperceptibility of the embedded watermark using the proposed algorithm. In order to evaluate the quality of watermarked signal, the following signal-to-noise ratio (SNR) equation is used:

$$
S N R=10 \cdot \log \frac{\sum_{i=0}^{N-1} s(i)^{2}}{\sum_{i=0}^{N-1}\left[s(i)-s^{w}(i)\right]^{2}}
$$

where $s(n)$ and $s^{w}$ (n) are original audio signal and watermarked audio signal respectively.

After embedding watermark, the SNR of all selected audio signals using the proposed method are above $25 \mathrm{~dB}$ (Table 2) which ensures the imperceptibility of the proposed system. Which satisfies the IFPI requirement $(20 \mathrm{~dB})$ described in [21]. These results have clearly demonstrated the superiority of the proposed scheme over Cox and Dhar methods.

Table 2. SNR comparison between proposed system , Cox's and Dhar's systems

\begin{tabular}{|l|c|c|c|c|}
\hline $\begin{array}{l}\text { Audio } \\
\text { signals }\end{array}$ & proposed & $\begin{array}{l}\text { DWT- } \\
\text { DCT }\end{array}$ & Dhar & Cox \\
\hline S1 & $\mathbf{2 8 . 9 1}$ & 26.90 & 21.81 & 17.23 \\
\hline S2 & $\mathbf{2 5 . 1 5}$ & 23.29 & 18.49 & 16.03 \\
\hline S3 & $\mathbf{2 5 . 8 9}$ & 23.63 & 21.07 & 16.23 \\
\hline S4 & $\mathbf{2 6 . 9 6}$ & 25.50 & 20.25 & 18.33 \\
\hline S5 & $\mathbf{3 1 . 0 7}$ & 29.44 & 19.07 & 16.63 \\
\hline S6 & $\mathbf{2 7 . 1 2}$ & 22.49 & 20.93 & 17.33 \\
\hline S7 & $\mathbf{2 7 . 1 3}$ & 23.99 & 20.71 & 16.74 \\
\hline S8 & $\mathbf{2 6 . 6 5}$ & 22.29 & 21.46 & 18.25 \\
\hline Mean & $\mathbf{2 5 . 5 1}$ & 22.06 & 19.92 & 14.77 \\
\hline
\end{tabular}

\section{IV.2 Robustness Test}

In order to evaluate the performance of the proposed watermarking system, the following Normalized Correlation (NC) is used to evaluate the correlation between the extracted watermark and the original watermark:

$$
N C\left(w, w^{\prime}\right)=\frac{\sum_{i=1}^{M_{1}} \sum_{j=1}^{M_{2}} w(i, j) w^{\prime}(i, j)}{\sqrt{\sum_{i=1}^{M_{1}} \sum_{j=1}^{M_{2}} w(i, j)^{2}} \sqrt{\sum_{i=1}^{M_{1}} \sum_{j=1}^{M_{2}} w^{\prime}(i, j)^{2}}}(11)
$$

Where $i$ is the index in the watermark image w. Table 3 shows the performance comparison in terms of $\mathrm{NC}$ between the proposed system and Cox's and Dhar's methods when no attack is applied to four different types of watermarked audio signals.

Table 3. Watermark detection result of the proposed system against other systems

\begin{tabular}{|l|l|l|l|l|}
\hline Audio signals & proposed & DWT-DCT & Dhar & Cox \\
\hline S1 & 1 & 1 & 1 & 1 \\
\hline S3 & 1 & 1 & 1 & 1 \\
\hline S5 & 1 & 1 & 1 & 1 \\
\hline S7 & 1 & 1 & 1 & 1 \\
\hline
\end{tabular}

Various common signal-processing attacks are used to assess the robustness of the proposed scheme are given below (Table 4).

(A) MP3 compression 64 kbps: The MPEG-1 layer-3 compression is applied. The watermarked audio signal is compressed at the bit rate of $64 \mathrm{kbps}$ and then decompressed back to the WAVE format.

(B) Resampling: The watermarked signal, originally sampled at $44.1 \mathrm{kHz}$, is resampled at $22.05 \mathrm{kHz}$, and then restored back by sampling again at $44.1 \mathrm{kHz}$. 
(C) Requantization: The 16-bit watermarked audio signal is re-quantized down to 8 bits/sample and then back to 16 bits/sample.

(D) Additive white Gaussian noise (AWGN):] White Gaussian noise is added to the watermarked signal until the resulting signal has an SNR of $15 \mathrm{~dB}$.

(E) Low-pass filtering: A second-order Butterworth filter with cut-off frequency $4 \mathrm{kHz}$ is used.

(F) Cropping: $10 \%$ segments are removed from the watermarked audio signal at the beginning and subsequently replaced by segments of the original signal.

(G) High-pass filtering: A second-order Butterworth filter with cut-off frequency $400 \mathrm{~Hz}$ is used.

(H) Amplification: watermarked audio signal is amplified by different amplification rate $(0.75 \%, 150 \%$, etc. $)$.

The presented algorithm is simulated by programming in Matlab 7.3. The size of the original signal S is $512 \times 1024$ and the size of image watermark $w$ is $32 \times 32$.

The watermark image $w$, and its finit ridgelte transform are shown respectively in Figure 4.

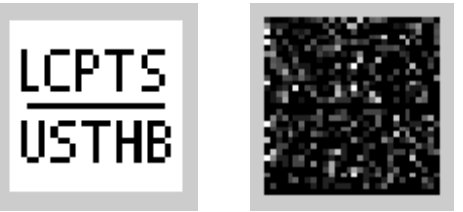

Fig 4: Original and encrypt image

The second image can be recoverd to original image after using the inverse FRIT transform with the keys generated in the insertion process as shown in Figure 2.

A comparaison between watermarks which were extracted after applying various attacks using the proposed algorithm, cox's algorithm and Dhar's algorithm are given in Figure 5. As shown in this figure, the quality of the extracted watermarks varies from one attack to another, however, the proposed technique is able to extract recognizable watermarks after all attacks, even in the case of high-pass filtering and amplification. While the cox's and Dhar's algorithms showed no resistance to these two attacks. So, they failed to preserve and detect the inserted watermark.

In addition to the extracted watermark quality and also the SNR of the watermarked audio signal which is described in the previous section, another privilege of the proposed method can be depicted in robustness performance especially in case of MP3 compression with different bit rates and Additif White Gaussian Noise with different SNR, as shown in Figures 6 and 7.
Table 4. Robustness comparison of proposed algorithm, Cox's and Dhar's algorithms

\begin{tabular}{|c|c|c|c|c|}
\hline & \begin{tabular}{|l|} 
Audio \\
signal \\
S
\end{tabular} & $\begin{array}{l}\text { Propose } \\
\text { d }\end{array}$ & $\begin{array}{l}\text { ALG } \\
2\end{array}$ & \begin{tabular}{|l} 
ALG \\
1
\end{tabular} \\
\hline \multirow{4}{*}{$\begin{array}{l}\text { MP3 } \\
\text { compression } \\
(64 \mathrm{kbps})\end{array}$} & S1 & \begin{tabular}{|l}
0.999 \\
\end{tabular} & \begin{tabular}{|l|}
0.971 \\
\end{tabular} & 0.960 \\
\hline & \begin{tabular}{|l|} 
S3 \\
\end{tabular} & \begin{tabular}{|l|}
0.999 \\
\end{tabular} & 0.966 & 0.975 \\
\hline & $\begin{array}{l}\text { S5 } \\
\end{array}$ & 0.999 & \begin{tabular}{|l|}
0.977 \\
\end{tabular} & 0.991 \\
\hline & \begin{tabular}{|l|} 
S7 \\
\end{tabular} & \begin{tabular}{|l|}
0.999 \\
\end{tabular} & 0.955 & 0.992 \\
\hline \multirow{4}{*}{$\begin{array}{l}\text { Resampling } \\
(44.1 \rightarrow 22.05 \rightarrow 44 \text {. } \\
1\end{array}$} & S1 & 1 & \begin{tabular}{|l|}
0.507 \\
\end{tabular} & 0.125 \\
\hline & S3 & 1 & \begin{tabular}{|l|}
0.507 \\
\end{tabular} & 0.152 \\
\hline & S5 & 1 & \begin{tabular}{|l|}
0.977 \\
\end{tabular} & 1 \\
\hline & S7 & 1 & 0.977 & 0.967 \\
\hline \multirow{4}{*}{$\begin{array}{l}\text { Requantification } \\
16 \rightarrow 8 \rightarrow 16\end{array}$} & S1 & 1 & 0.961 & 0.991 \\
\hline & S3 & 1 & 0.977 & 0.999 \\
\hline & \begin{tabular}{|l|} 
S5 \\
\end{tabular} & 1 & \begin{tabular}{|l|}
0.977 \\
\end{tabular} & 1.000 \\
\hline & \begin{tabular}{|l|} 
S7 \\
\end{tabular} & 1 & 0.973 & 0.999 \\
\hline \multirow{4}{*}{$\begin{array}{l}\text { Additive White } \\
\text { Gaussian Noise } \\
(20 \mathrm{~dB})\end{array}$} & S1 & \begin{tabular}{|l|}
0.999 \\
\end{tabular} & \begin{tabular}{|l|}
0.977 \\
\end{tabular} & 0.819 \\
\hline & S3 & 0.999 & \begin{tabular}{|l|}
0.977 \\
\end{tabular} & 0.978 \\
\hline & S5 & \begin{tabular}{|c|}
0.998 \\
\end{tabular} & \begin{tabular}{|l|}
0.977 \\
\end{tabular} & 0.997 \\
\hline & \begin{tabular}{|l|} 
S7 \\
\end{tabular} & 0.999 & 0.976 & 0.996 \\
\hline \multirow{4}{*}{$\begin{array}{l}\text { Low-pass } \\
\text { filtering } \\
(\mathrm{fc}=4 \mathrm{kHz})\end{array}$} & S1 & 1 & 0.856 & 0.437 \\
\hline & \begin{tabular}{|l|} 
S3 \\
\end{tabular} & 1 & \begin{tabular}{|l}
0.977 \\
\end{tabular} & 0.987 \\
\hline & \begin{tabular}{|l|} 
S5 \\
\end{tabular} & 1 & 0.977 & 0.983 \\
\hline & \begin{tabular}{|l|} 
S7 \\
\end{tabular} & 1 & 0.960 & 0.982 \\
\hline \multirow{4}{*}{$\begin{array}{l}\text { Cropping } \\
(10 \%)\end{array}$} & S1 & \begin{tabular}{|l|}
0.995 \\
\end{tabular} & 0.977 & 0.999 \\
\hline & S3 & $\begin{array}{l}0.994 \\
\end{array}$ & \begin{tabular}{|l|}
0.977 \\
\end{tabular} & 0.999 \\
\hline & S5 & 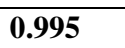 & 0.977 & \begin{tabular}{|l|}
0.999 \\
\end{tabular} \\
\hline & \begin{tabular}{|l|} 
S7 \\
\end{tabular} & \begin{tabular}{|l|}
0.995 \\
\end{tabular} & 0.977 & 0.999 \\
\hline \multirow{4}{*}{$\begin{array}{l}\text { High-pass } \\
\text { filtering } \\
(\mathrm{fc}=400 \mathrm{~Hz})\end{array}$} & S1 & 1 & 0.503 & 0.777 \\
\hline & \begin{tabular}{|l|} 
S3 \\
\end{tabular} & 1 & 0.376 & 0.810 \\
\hline & S5 & 1 & 0.528 & 0.810 \\
\hline & \begin{tabular}{|l|} 
S7 \\
\end{tabular} & 1 & 0.346 & 0.808 \\
\hline \multirow{4}{*}{$\begin{array}{l}\text { Amplification } \\
(150 \%)\end{array}$} & S1 & \begin{tabular}{|l|}
0.992 \\
\end{tabular} & 0.846 & 0.491 \\
\hline & S3 & 0.992 & 0.846 & 0.491 \\
\hline & $\begin{array}{l}\text { S5 } \\
\end{array}$ & \begin{tabular}{|l|}
0.992 \\
\end{tabular} & 0.846 & 0.491 \\
\hline & \begin{tabular}{|l|} 
S7 \\
\end{tabular} & \begin{tabular}{|l|}
0.992 \\
\end{tabular} & 0.846 & 0.491 \\
\hline
\end{tabular}

(a)

(b)

(c)

(d)

\section{LLFTS LLFTS LLFTS LLFTS DSTHE DSTHE DJTHE DSTHE}

(e)

$\frac{\text { LIFTS LEPTS }}{\text { USTHE }} \frac{\text { LEFTS }}{\text { USTHE }}$ (h) (i) 
(a)

(b)

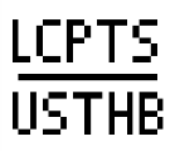

(e)

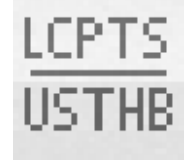

(a)

\section{LEFTS}

(e)

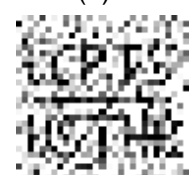

+2 ing

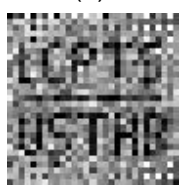

(f)

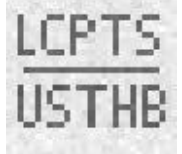

(ii) (c)

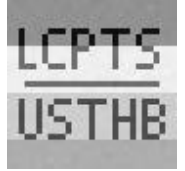

(g)

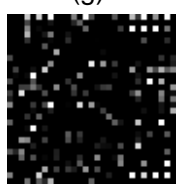

(ii) (d)

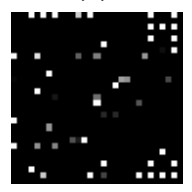

(h)

:.:

(d) $\cdots \cdot$ (c)

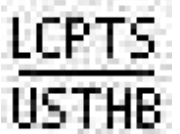

(g)

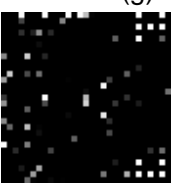

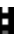

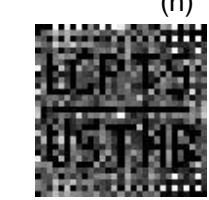

(iii)

Fig 5: Extracted watermarks using: i) proposed algorithm, ii) cox's algorithm, iii) Dhar's algorithm after different attacks a) original watermark, b) MP3 compression, c) requantization, d) resampling, d) AWGN, e) low pass filtering, f) High-pass filtering, g) amplification

It is clear from Figures 6 and 7, the efficiency of the proposed algorithm to preserve the watermarks inserted into the audio signals to prove the copyright protection. It shows a high superiority against other algorithms of comparison.

\section{CONCLUSION}

A novel audio watermarking method is proposed based on double embedding in the hybrid domain, DWT-DST. The method uses the DWT variety of time-frequency decomposition for audio signals, and modifies its DST coefficients with a watermark image before re-constituting the signal. The effectiveness of the DWT-DST based algorithm was demonstrated by watermarking eight different audio signals. Moreover, it is also a secure scheme, the watermark cannot be extracted without knowing the keys generated during the insertion process.

Future work will concentrate on making the method more practical by modifying the technique such that the original audio signal is not required to extract the watermark and this approach may also be extended to image and video watermarking.

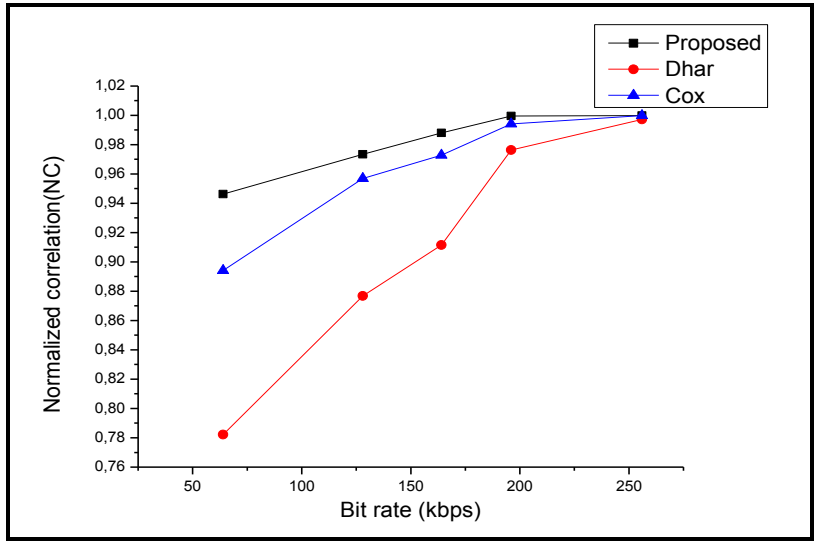

Fig 6: Comparison of robustness against MP3 compression with different bit rates

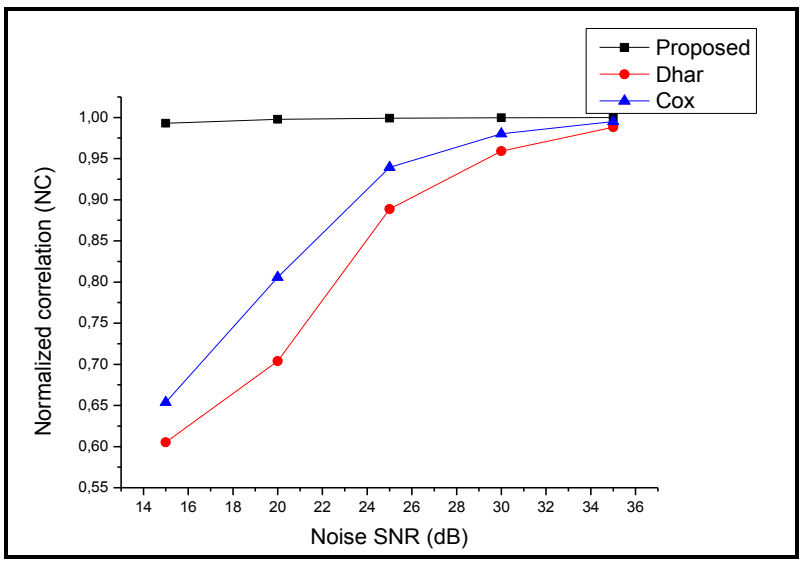

Fig 7: Comparison of robustness against AWGN with different SNR

\section{REFERENCES}

[1] Petitcolas, F.A.P, Anderson, R.J, Kuhn, M.G , "Information hiding-a survey," Proceedings of the IEEE, Vol 87 , No. 7, pp. 1062 - 1078, 1999

[2] J. X. Liu, Z. M. Lu ; J. S. Pan "A Robust Audio Watermarking Algorithm Based on DCT and Vector Quantization," Eighth International Conference on Intelligent Systems Design and Applications, 2008.

[3] M. Arnold, "Audio watermarking: features, applications and algorithms," in Proc. of IEEE International Conference on Multimedia and Expo, vol. 2, pp. 10131016,2000

[4] P. Bassia, I. Pitas, N. Nikolaidis, "Robust Audio Watermarking in the Time Domain". IEEE Transactions on Multimedia, vol 3, No. 2, 2001.

[5] W. Bender, D.Gruhl, and N. Morimoto, "Techniques for data hiding, ” IBM Systems journal, 1996, 35(3/4): 131336.

[6] J. Huang, Y. Wang, and Y.Q. Shi, "A blind audio watermarking algorithm with self-synchronization," Proc. Of IEEE, Int. Sym. On Circuits and Systems, vol. 3 pp. 627-630, 2002. 
[7] D. Gruhl, A. Lu and W. Bender, "Echo Hiding," Proc. Of $1^{\text {st }}$ Information Hiding Workshop, LNCS vol. 1174, Berlin, Germany: Springer - Verlag, pp. 295 -315, 1996.

[8] Kim, H.J., and Choi Y.H., "A novel echo-hiding scheme with backward and forward kernels," IEEE Transactions on circuits and Systems for video and technology, vo1. 13, 2003.

[9] A.N. Lemma, J. Aprea, W. Oomen, L.v. Kerkhof, "A temporal domain audio watermarking technique, " IEEE Trans. Signal Process., 51 (4) (2003), pp. 1088-1097.

[10] Boney, L., Tewfik, A. H., and Hamdy, K. N., "Digital watermarks for audio signal," International Conference on Multimedia Computing and Systems, pp. 473-480, 1996.

[11] I. J. Cox, J. Kilian, F. T. Leighton, and T. Shamoon. "Secure spread spectrum watermarking for multimedia. IEEE Trans. Vol. 6, No. 12, Dec 1997.

[12] P. K. Dhar, M. I. Khan, C. H. Kim, and J. M. Kim, "An Efficient Audio Watermarking Algorithm in Frequency Domain for Copyright Protection, " CCIS, 2010, Vol 122, pp. 104-113, Springer

[13] Michael A., Gerzon and peter G. Graven., "A high-rate buried-data channel for audio CD", Journal of the Audio Engineering Society, 43(1/2):3-22, January - February 1995.

[14] Shaoquan Wu, J. Huang, Daren Huang, Y.Q. Shi, "SelfSynchronized Audio Watermark in DWT Domain", IEEE (ISCAS), v-712 -v-715, 2004
[15] N. Sriyingyong, and K. Attakitmongcol "Wavelet-Based Audio Watermarking Using Adaptive Tabu Search" Wireless Pervasive Computing, 2006 1st International Symposium on 16-18 an. 2006 Page(s):1 - 5, 2006.

[16] I.Daubechies "Orthonormal Bases of Compactly Supported Wavelets," Communications on Pure and Applied Math. Vol.41 1988, 909-996.

[17] R. M. Zhao, H. Lian, H. W. Pang, B.N. Hu, "A Watermarking Algorithm by Modifying AC Coefficies in DCT Domain," International Symposium on Information Science and Engieering, pp. 159-162. IEEE, 2008.

[18] L. Yaroslavsky, Y. Wang, "DFT, DCT, MDCT, DST and signal Fourier spectrum analysis," 10th European Signal Processing Conference, pp. 1065-1068, Tampere, Finland 2000 .

[19] D. Salomon. G. Motta and D. Bryant, "Data Compression: The Complete Reference," Fourth Edition. Springer-Verlag 2007.

[20] Minh N. Do, Martin Vetterli, "The Finite Ridgelet Transform for Image Representation, " IEEE Trans on Image Processing, Vol. 12, No. 1, pp. 16 - 28, 2003.

[21] S. Wu, J. Huang, D. Huang, Y. Q. Shi, "Efficiently SelfSynchronized Audio Watermarking for Assured Audio Data Transmission, " IEEE Trans on Broadcasting, Vol 51, No. 1, pp. $69-76,2005$ 